\title{
A novel bladder cancer urinary biomarker: can it go where no marker has gone before?
}

\author{
Ryan W. Dobbs, Michael R. Abern \\ Department of Urology, The University of Illinois at Chicago, Chicago, IL, USA \\ Correspondence to: Michael R. Abern, MD. Department of Urology, the University of Illinois at Chicago, 820 S. Wood Street Suite 515, Chicago, IL \\ 60612, USA. Email: Mabern1@uic.edu. \\ Provenance: This is a Guest Editorial commissioned by Section Editor Xiao Li (Department of Urologic Surgery, The Affiliated Cancer Hospital of \\ Jiangsu Province of Nanjing Medical University, Nanjing, China). \\ Comment on: Pichler R, Fritz J, Tulchiner G, et al. Increased accuracy of a novel mRNA-based urine test for bladder cancer surveillance. BJU Int \\ 2018;121:29-37.
}

Submitted Dec 18, 2017. Accepted for publication Jan 03, 2018.

doi: $10.21037 /$ tau.2018.01.04

View this article at: http://dx.doi.org/10.21037/tau.2018.01.04

In their recent publication in BJUI, Pichler et al. (1) report the diagnostic accuracy of Xpert BC Monitor, a biomarker combining the measured expression of 5 mRNAs (ABL1, CRH, IGF2, UPK1B \& ANXA10) in voided urine. The study evaluated the diagnostic accuracy of the assay in 140 patients with a known history of NMIBC. White light cystoscopy and urine cytology were the diagnostic gold standard. Within this cohort, 43 patients (30.7\%) were found to have a tumor recurrence. The overall sensitivity (0.84) and negative predictive value (0.93) of the Xpert BC Monitor were significantly higher than those of bladder washing cytology $(0.33$ and 0.76 respectively; $\mathrm{P}<0.001)$ Subgroup analyses demonstrated a sensitivity of $100 \%$ for patients with high-grade tumors with Xpert BC Monitor as compared to $83 \%$ of bladder washing cytology. Xpert BC Monitor also outperformed bladder washing cytology with significantly higher sensitivities in low grade (77\% vs. $13 \%)$, Ta ( $82 \%$ vs. $21 \%)$, unifocal (68\% vs. $18 \%$ ) and low volume $(<3 \mathrm{~cm})$ disease $(75 \%$ vs. $29 \%)$.

The excellent diagnostic accuracy of Xpert BC Monitor in this study warrants external validation. We have shown previously (2) that a urinary bladder cancer biomarker with thresholds optimized for a development cohort may not validate as well in other cohorts. There are several causes for failure of external validation studies to achieve the accuracy of the development study, even if internal validation is performed. Variance within the techniques of measurement and instrumentation, in this case mRNA quantification, or handling and processing of the biospecimens may lead to differing accuracy. In addition, selection bias of the study cohort results in differences in measured or unmeasured patient characteristics and probability of disease and therefore differences in the performance of the test. The cohort of the Pichler study does appear representative of the general bladder cancer population with regard to severs measures characteristics such as gender, age, tumor characteristics, and exposure to intravesical therapy, for which the authors should be commended. However an external validation study is the only way to decipher the presence of unmeasured selection bias.

As such, we suggest several design considerations for an external validation study. Importantly, the histopathologic confirmation should be included as an additional gold standard to test the accuracy of the Xpert BC Monitory. Recent introductions of cystoscopy adjuncts such as blue light hexaminolevulinate (3) and narrow band imaging (NBI) (4) could provide additional data for the diagnostic gold standard as these technologies have been increasingly incorporated into clinical practice. This will more accurately characterize false positives from white light cystoscopy and false negatives when random biopsies return positive. In addition, a larger cohort of patients with prior BCG therapy would be useful, as this is a known confounder of conventional urinary cytology. Finally, an analysis of clinical utility should be performed using a decision curve (5) or other methodology. The authors reported a high rate of negative internal control signal necessitating repeat Xpert BC Monitor analysis in 15 patients (10.7\%) which may limit 
its utility. The lack of consideration for clinical utility is a major reason for the lack of routine use of the several FDA approved urinary biomarkers that have increased diagnostic accuracy compared to urinary cytology. The relatively low morbidity of the gold standard cystoscopy creates a high bar for a test to supplant its use (6).

If the excellent accuracy of the Xpert BC Monitor test reported by Pichler et al. can be repeated in a well-designed external validation study it could provide several clinical benefits. Due to its natural history of frequent recurrences and need for long periods of surveillance, bladder cancer is the costliest of any malignancy $(7,8)$. Adding the Expert BC Monitor could drive cost down if the negative predictive value were high enough to use it to reduce the frequency or duration of cystoscopic surveillance. If the specificity were excellent, cystoscopy could be used as a reflex test thereby reserving the invasive procedure to those with high risk of a tumor.

\section{Acknowledgements}

None.

\section{Footnote}

Conflicts of Interest: The authors have no conflicts of interest to declare.

\section{References}

1. Pichler R, Fritz J, Tulchiner G, et al. Increased accuracy

Cite this article as: Dobbs RW, Abern MR. A novel bladder cancer urinary biomarker: can it go where no marker has gone before? Transl Androl Urol 2018;7(Suppl 1):S96-S97. doi: $10.21037 /$ tau.2018.01.04 of a novel mRNA-based urine test for bladder cancer surveillance. BJU Int 2018;121:29-37.

2. Fantony JJ, Abern MR, Gopalakrishna A, et al. Multiinstitutional external validation of urinary TWIST1 and NID2 methylation as a diagnostic test for bladder cancer. Urol Oncol 2015;33:387.e1-6.

3. Grossman HB, Gomella L, Fradet Y, et al. A phase III, multicenter comparison of hexaminolevulinate fluorescence cystoscopy and white light cystoscopy for the detection of superficial papillary lesions in patients with bladder cancer. J Urol 2007;178:62-7.

4. Herr HW, Donat SM. A comparison of white-light cystoscopy and narrow-band imaging cystoscopy to detect bladder tumour recurrences. BJU Int 2008;102:1111-4.

5. Vickers AJ, Elkin EB. Decision curve analysis: a novel method for evaluating prediction models. Med Decis Making 2006;26:565-74.

6. Biardeau X, Lam O, Ba V, et al. Prospective evaluation of anxiety, pain, and embarrassment associated with cystoscopy and urodynamic testing in clinical practice. Can Urol Assoc J 2017;11:104-10.

7. Canter DJ, Revenig LM, Smith ZL, et al. Re-examination of the natural history of high-grade T1 bladder cancer using a large contemporary cohort. Int Braz J Urol 2014;40:172-8.

8. Botteman MF, Pashos CL, Redaelli A, et al. The health economics of bladder cancer: a comprehensive review of the published literature. Pharmacoeconomics 2003;21:1315-30. 